\title{
Persistent Postprandial Angina in a Patient With Gastroesophageal Reflux Disease: A Diagnostic Dilemma
}

\author{
Joshua K. Salabei ${ }^{1}$, Troy J. Fishman ${ }^{1}$, Zekarias T. Asnake ${ }^{1}$, Matthew Calestino ${ }^{2}$ \\ 1. Internal Medicine, University of Central Florida College of Medicine, Hospital Corporation of America North Florida \\ Division, Gainesville, USA 2. Internal Medicine, North Florida Regional Medical Center, University of Central Florida, \\ Gainesville, USA
}

Corresponding author: Joshua K. Salabei, joshua.salabei@hcahealthcare.com

\begin{abstract}
Chest pain (CP) is a common reason for visits to the emergency department (ED). The underlying etiology of a good number of cases of $\mathrm{CP}$ can be diagnosed with adequate history taking and routine laboratory testing. However, atypical presentations of $\mathrm{CP}$, in the settings of other causes of $\mathrm{CP}$ such as gastroesophageal reflux disease (GERD), can sometimes be tricky to diagnose with only routine lab tests and electrocardiogram (EKG). Herein, we present a 73-year-old male with a history of GERD and coronary artery disease who presented to our ED complaining of postprandial CP unaffected by exertion or rest. Initially, his symptoms were thought to be GERD-related but other heart-related causes of $\mathrm{CP}$ were considered due to the persistence of his CP postprandially. A cardiac stress test was subsequently done to rule out possible cardiac causes of his CP. His stress test was abnormal prompting heart catheterization that showed almost complete occlusion of his left anterior descending (LAD) and left circumflex (LCx) arteries. His symptoms resolved post-catheterization/stenting of his LAD and LCx arteries. He was later discharged unconditionally. His presentation highlights the required vigilance physicians must maintain when interrogating $\mathrm{CP}$, even when other non-cardiac-related causes seem more plausible.
\end{abstract}

Received 06/02/2020 Review began 06/21/2020 Review ended 08/11/2020 Published 08/16/2020

○) Copyright 2020

Salabei et al. This is an open access article distributed under the terms of the Creative Commons Attribution License CC-BY 4.0., which permits unrestricted use, distribution, and reproduction in any medium, provided the original author and source are credited.
Categories: Cardiac/Thoracic/Vascular Surgery, Cardiology, Internal Medicine

Keywords: coronary steal, angiography, coronary artery disease, post-prandial pain, angina, pci, case report

\section{Introduction}

Chest pain (CP) is a common reason for visits to the emergency department (ED). Multiple etiologies for CP have been described in the literature, and a good number of these etiologies can be diagnosed with adequate history taking and routine laboratory testing [1,2]. However, atypical presentations of CP, in the settings of other causes of CP such as gastroesophageal reflux disease (GERD), can sometimes be tricky to diagnose with only routine evaluations. For example, a patient presenting with postprandial CP unaffected by exertion/rest can be easily misdiagnosed as a non-cardiac CP, especially if initial evaluation involving troponin measurements and electrocardiogram (EKG) are unremarkable. Such misdiagnosis can be fatal; therefore, physicians are cautioned to be on the guard when interrogating $\mathrm{CP}$ even when other non-cardiac causes seem more plausible. This case highlights the importance of objective assessment of CP especially in patients with a history of coronary artery disease (CAD).

\section{Case Presentation}

We present the case of a 73-year-old man with a past medical history of CAD with a stent in the right coronary artery (RCA) placed seven years earlier and chronic GERD with Barrett's esophagus, who presented to our ED complaining of a dull non-radiating CP for several weeks. He was lost to follow-up after the placement of his RCA stent. He described his CP as episodic, occurring postprandially, and unaffected by strenuous activity. Pain is not associated with palpitations or diaphoresis. On physical exam, the pain was non-reproducible. The remainder of his physical exam was also non-contributory. Upon presentation to the ED, serial troponins were within normal limits and his EKG showed no ST elevations (Figure 1). 


\section{Cureus}

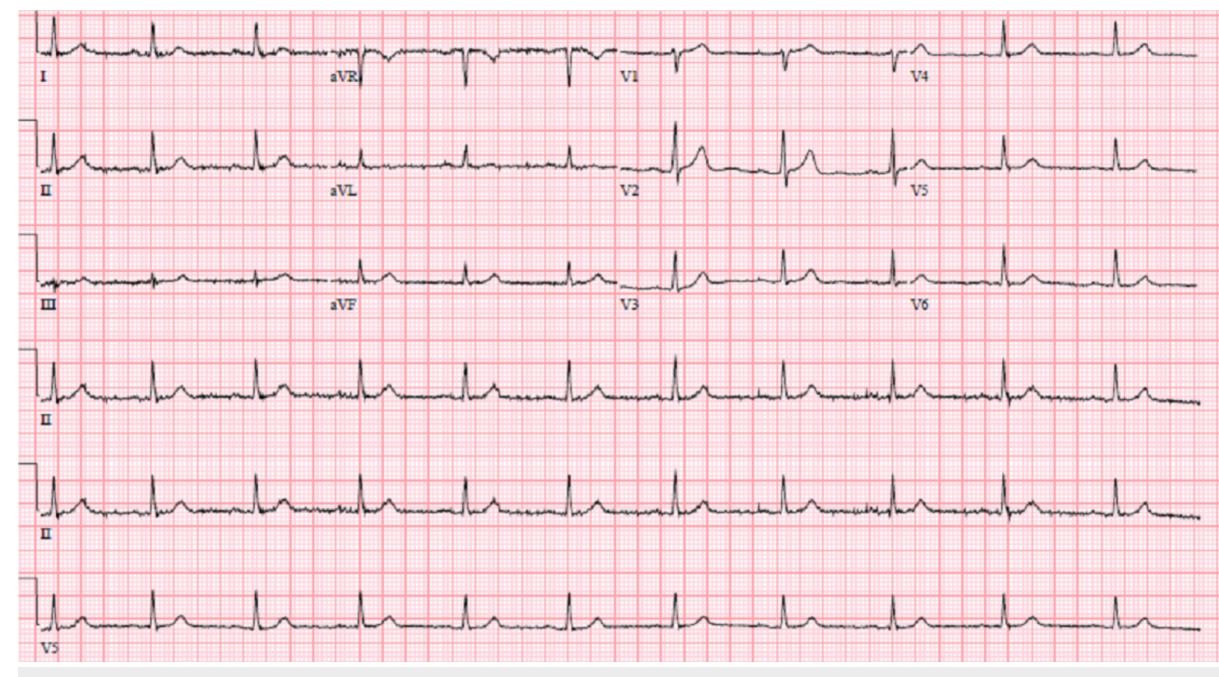

FIGURE 1: Electrocardiogram at presentation.

His other laboratory tests are shown in Table 1.

\begin{tabular}{|c|c|c|}
\hline Test & Value on Admission & Reference \\
\hline Hemoglobin & $15.2 \mathrm{~g} / \mathrm{dL}$ & $12.0-15.0 \mathrm{~g} / \mathrm{dL}$ \\
\hline WBC & 9.8 thou $/ \mathrm{mm}^{3}$ & $4.5-11.0$ thou $/ \mathrm{mm}^{3}$ \\
\hline Alkaline phosphatase & 54 units/L & 46-116 units/L \\
\hline ASI & 8 units/L & $15-3 /$ units/L \\
\hline ALT & 17 units/L & 16-61 units/L \\
\hline Iroponins & $0.019 \mathrm{ng} / \mathrm{mL}$ & $<0.05 \mathrm{ng} / \mathrm{mL}$ \\
\hline
\end{tabular}

\section{TABLE 1: Laboratory results at the time of presentation}

WBC: white blood cell count, AST: aspartate aminotransferase, ALT: alanine aminotransferase

Upper abdominal ultrasound was significant for cholelithiasis with sludge and a thickened gallbladder wall of $4 \mathrm{~mm}$ without sonographic Murphy's sign (Figure 2A, 2B). He was treated with pantoprazole and provided with other supportive measures. Plans for elective cholecystectomy were made. However, he continued to experience postprandial $\mathrm{CP}$ while in the hospital, prompting further evaluation. Owing to his history of $\mathrm{CAD}$ with a stent and lack of follow-up since first stent placement, suspicion for postprandial angina became high on the differentials. Therefore, an echocardiogram was performed, and it showed an ejection fraction of 55\%-60\% without wall motion abnormalities. A myocardial perfusion scan (Regadenoson nuclear stress test) showed ischemia in the mid-basal inferolateral wall (Figure 2C). 


\section{Cureus}
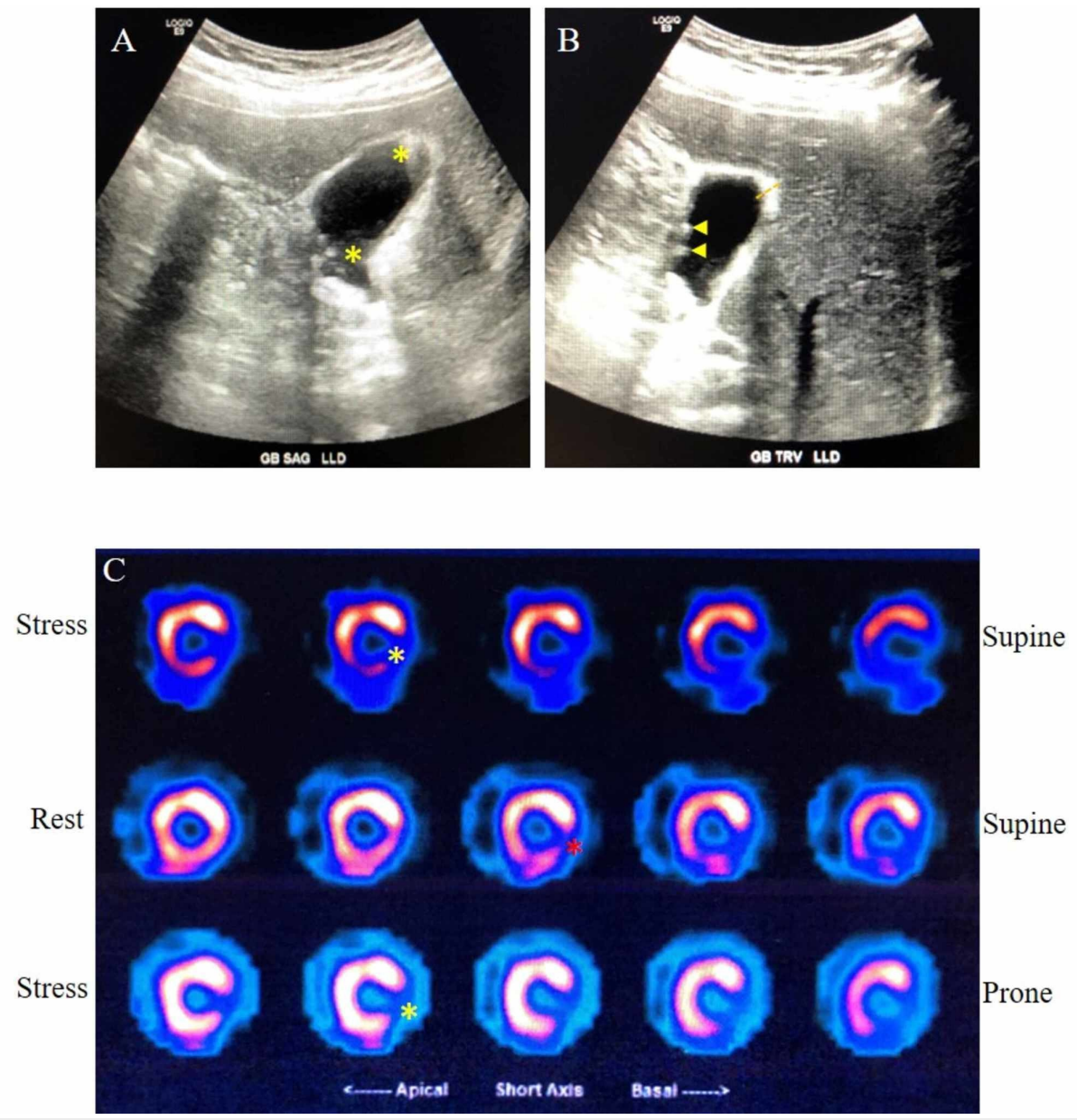

FIGURE 2: Representative gallbladder and myocardial perfusion scan images

(A) Sagittal view of gallbladder (GB) showing sludge (yellow asterisks). (B) Transverse view of GB showing gallstones indicated with yellow arrowheads, and thickened GB wall indicated with the orange line. (C) Representative myocardial perfusion scan images. Reversible (yellow asterisks) and irreversible changes (red asterisk) after stress are noted. Irreversible areas represent ischemic regions from prior myocardial infarct.

Subsequent coronary catheterization revealed severe CAD with 100\% occlusion of the left circumflex (LCX) and $90 \%$ occlusion of the left anterior descending (LAD) arteries (Figure 3). Both stenosed arteries were successfully revascularized with stents, leading to a complete resolution of his postprandial symptoms. He was then discharged on aspirin and clopidogrel and was advised to follow up with his cardiologist and primary care physician 


\section{Cureus}
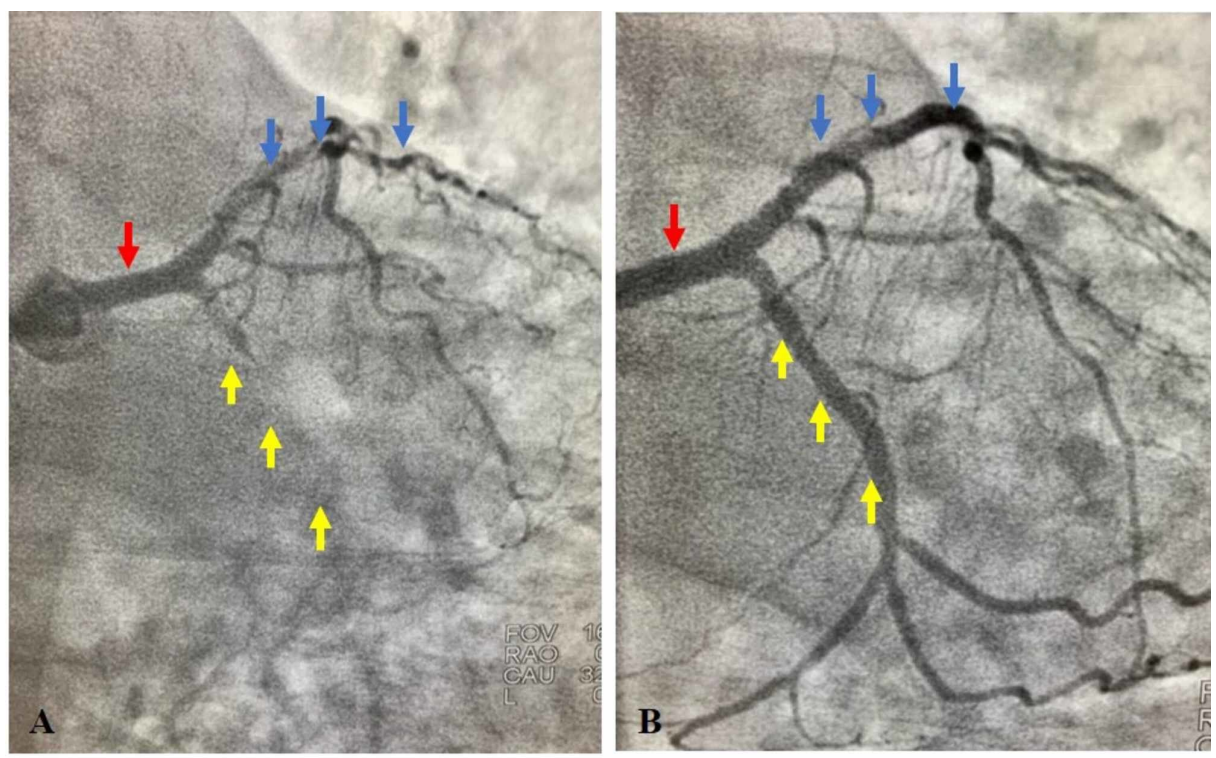

FIGURE 3: Coronary perfusion scan before and after revascularization

(A) Coronary perfusion scan before revascularization. The red arrow shows the LCA with its two main branches: the LCX and the LAD. The yellow arrows indicate the presumed track of the LCX artery. The blue arrows indicate the LAD, showing severe stenosis in its subsequent downstream branches. (B) Postrevascularization. The red arrow shows the LCA with its two main branches; the LCX and the LAD. The yellow arrows indicate the LCX artery, showing restoration of blood flow. The blue arrows indicate the LAD, also showing restoration of blood flow to its downstream branches. LCA; left coronary artery, LAD; left anterior descending artery, LCX; left circumflex artery

\section{Discussion}

With CAD among the main causes of death in the USA and worldwide, physicians must recognize subtle and atypical presentations of cardiac-related causes of $\mathrm{CP}$ that could be detrimental if missed [3]. Our case highlights this in that (I) the patient had a history of chronic GERD with Barrett's esophagus, (II) his CP was only exacerbated postprandially, and (III) abdominal ultrasound was significant for cholelithiasis and gallbladder wall thickening. Thus, his history and ultrasound findings, if not interpreted with caution, could serve as potential distractors from the actual cause of his symptoms. Therefore, especially in patients with a history of CAD, any form of $\mathrm{CP}$, typical or atypical, must be interrogated with caution.

The patient had complete occlusion of the LCX and 90\% occlusion of the LAD arteries (Figure 2). With this degree of occlusion, it is surprising that his symptoms were not exacerbated by strenuous activity, but rather only postprandially. One possible explanation for this is that the fully patent RCA was sufficient, in addition to collateral circulation, to supply the entire left ventricle.

Over the last couple of years, it has been increasingly recognized that blood supply via redistribution of myocardial blood flow could be the main cause of postprandial angina. For example, contrasting increased myocardial oxygen demand as a mechanism of postprandial angina, a study, using positron emission tomography and high-frequency pulse-wave Doppler echocardiography to measure coronary blood flow in the LAD coronary artery, demonstrated that myocardial blood flow decreases in the territory supplied by stenotic coronary arteries postprandially [2-4]. Still, the exact mechanism by which our patient's symptoms were exacerbated postprandially remains unanswered and although a postprandial EKG could have helped confirm blood flow compromise to the myocardium, it would have been insufficient to delineate a mechanism.

The sonographic findings of cholelithiasis and gallbladder wall thickening could have been potential distractors to pursuing a cardiac-related etiology of the patient's presenting symptom. In addition, he had a chronic history of GERD with Barret's esophagus, further serving as distractors. His EKG and trended troponins were all unremarkable, and his echocardiographic findings showed no wall abnormalities. Still, he continued to experience postprandial pain despite all the negative findings. The decision to further evaluate the patient was driven principally by objective clinical judgment, highlighting that each case is unique and must be approached as such. We have presented an atypical presentation of postprandial angina and highlight that $\mathrm{CP}$, especially in patients with a known history of CAD, must be approached with caution.

\section{Conclusions}

In this report, we have presented an atypical presentation of $\mathrm{CP}$ in a patient who has a known history of 
GERD. We have highlighted that in patients with a significant history of cardiovascular disease, CP must be interrogated fully, irrespective of the presence of other obvious causes of CP, such as GERD. Since CP is one of the most leading reasons for hospital visits, it is important that physicians be aware of atypical presentations such as presented here. Objective judgment must be applied on a case-by-case basis.

\section{Additional Information}

\section{Disclosures}

Human subjects: Consent was obtained by all participants in this study. Conflicts of interest: In compliance with the ICMJE uniform disclosure form, all authors declare the following: Payment/services info: All authors have declared that no financial support was received from any organization for the submitted work. Financial relationships: All authors have declared that they have no financial relationships at present or within the previous three years with any organizations that might have an interest in the submitted work. Other relationships: All authors have declared that there are no other relationships or activities that could appear to have influenced the submitted work.

\section{Acknowledgements}

This research was supported (in whole or in part) by HCA Healthcare and/or an HCA healthcare affiliated entity. The views expressed in this publication represent those of the author(s) and do not necessarily represent the official views of HCA Healthcare or any of its affiliated entities.

\section{References}

1. Chung WY, Sohn DW, Kim YJ, Oh S, Chai IH, Park YB, Choi YS: Absence of postprandial surge in coronary blood flow distal to significant stenosis: a possible mechanism of postprandial angina. J Am Coll Cardiol. 2002, 40:1976-1983. 10.1016/s0735-1097(02)02533-0

2. Baliga RR, Rosen SD, Camici PG, Kooner JS: Regional myocardial blood flow redistribution as a cause of postprandial angina pectoris. Circulation. 1998, 97:1144-1149. 10.1161/01.cir.97.12.1144

3. Benjamin EJ, Muntner P, Alonso A, et al.: Heart disease and stroke statistics-2019 update: a report from the American Heart Association. Circulation. 2019, 139:e56-e528. 10.1161/CIR.0000000000000659

4. Werner GS, Figulla HR: Direct assessment of coronary steal and associated changes of collateral hemodynamics in chronic total coronary occlusions. Circulation. 2002, 106:435-440.

10.1161/01.cir.0000022848.92729.33 